\title{
Genotypic and 20-week body weight effects on full sexual maturity traits of parent stock layers raised in commercial deep-litter system
}

\author{
Jesuyon Oluwatosin M.A. ${ }^{* 1}$ and Olawumi S.0. ${ }^{2}$ \\ ${ }^{*}$ Corresponding author: dr.oluwatosinjesuyon14@gmail.com
}

${ }^{1}$ Animal Breeding and Genetics Unit, Animal Production and Health Department. Federal University Oye-Are Road, P. M. B. 373, Oye - Ekiti, Ekiti State, Nigeria.

${ }^{2}$ Animal Science Department, Ekiti State University, Ado-Ekiti. E-mail: olawumisimeon@yahoo.com

\section{ABSTRACT}

The influence of genotype (GTY) and 20-week body weight (PW20) on full-sexual maturity (FSM) productivity and hatching traits were investigated on two parent-stock layers in Ibadan, Nigeria. Data were obtained on twentytwo batches of BOVAN NERA (BN) and ISA BROWN layers (IB) each, between 2009-2019, from a breeding company in Ibadan. These were grouped based on strain and 20-week body weight. Experimental design was randomized block design (RBD). Data were subjected to general linear model (GLM) factorial and Tukey's HSD, $\alpha=0.05$, procedures of Statistical Analytical Systems ${ }^{\circledR}$ (SAS, 2002) software. BN had better pullet weight $(\mathrm{P}<0.05$, $\mathrm{ds}=0.88)$, while IB possessed higher hen-day egg production, persistency of egg production and pullet day-old chicks hatched $(\mathrm{P}<0.05, \mathrm{ds}=-0.86$ to -1.44$)$ at FSM. The 20 -week low weight birds required intensive skillful management for compensatory weight-gain to FSM, while egg weight was higher in 20-week high weight class at full maturity $(\mathrm{P}<0.05$, ds $=0.06$ to -1.19$)$. Genotype by 20 -week pullet-weight interaction revealed better genetics of $\mathrm{BN}$ genotype on compensatory growth between 20th week and FSM, and on PFM ( $\mathrm{P}<0.05$, ds=0.24 to 1.62). The better HDF and P80 $(\mathrm{P}<0.05$, ds $=-1.73,-1.61)$ of medium weight IB genotype signals its genetic ability for higher percent day-old chicks production $(\mathrm{P}>0.05$, ds $=-0.72$ to -1.11$)$.

Keywords: Bovan nera, egg weight, Isa brown, persistency of egg production, pullet-chicks hatched. 


\section{INTRODUCTION}

Modern strains of hybrid layers such as Bovans, Hisex, Hy-Line, Lohman, H\&N, Isa, Starcross, Babcock, Shaver, etc are exotic and are imported to tropical countries to provide major source of hatchable and table eggs. Pullet weight is an important economic trait in layers, moderately heritable and influenced by the environment. Many works have been published on the benefits of body weight and age on sexual maturity and production traits in pullets (Connie et al., 1985, Tesfaye et al, 2009). Olawumi (2011) reported strain effects on 20-week body weight, but literature is limiting on the effect of 20 -week pullet weight on layer-breeder productivity and hatching traits at FSM. The full sexual maturity (FSM) point was taken as the peak of egg production by a flock of birds in lay, while the characteristics of a flock at this point determine subsequent laying behaviour and pattern of lay. From the stand-point of commercial breeding, the 20-week pullet weight could have important effect on FSM traits, and thus an important indicator for breeding and management of layer breeders before FSM. At 20-weeks, egg laying has commenced but may not be set into commercial incubator until an appropriate weight $(\geq 56 \mathrm{~g})$, usually obtained at about 23-25 weeks. Pullet weight also has positive effect on egg size (Duncan and Mench, 2000; Haq et al., 2011). Variation in pullet weight within flocks, strains and breeds is attributed to genetic variation and environmental factors that affect individuals (Ayorinde and Oke, 1995). Olawumi (2014) showed that pullet weight has direct and positive effect on laying capacity between and within breeds reared in same or different environments. In guinea fowl, Oke et al. (2004) reported the positive effect of body weight on egg number, egg weight, yolk weight and albumen weight. Excessive weight due to fat deposition could be problematic, leading to prolapse, drop in egg production and high mortality. Study was conducted to assess the influence of genotype and 20week pullet weight on egg productivity and hatching traits at FSM in Bovan Nera and Isa Brown layer breeders.

\section{MATERIALS AND METHODS}

Records were obtained from a reputable commercial breeder farm and hatchery in Ibadan, South-west Nigeria on twenty-four batches of BOVAN NERA (BN) and twenty batches of ISA BROWN (IB) parent stock layers, from 2009 to 2019. Data on 20-week body weight and peak egg production point, that represent attainment of full sexual maturity were collected from each batch of genotype that had about 3986 pullets and 600 cocks at point of lay. Each batch of a genotype was treated as a replicate of each other. Data were 
collected on twelve (12) productive and hatching traits namely pullet weight at 20-weeks (PW20, g), weight gain from 20 weeks to full maturity (WGF, g), feed intake at 20-weeks to full maturity(F20F, kg), feed intake at full maturity (FFM, g), age at full maturity (AFM, days), pullet weight at full maturity (PFM, g), hen-day production at full maturity (HDF, \%), egg weight at full maturity (EWF, g), persistency of egg production at $\geq 80 \%$ HDP at full maturity (P80, weeks), eggs-set at full maturity (ESF, \%), fertility of eggs-set at full maturity (FES, \%), hatchability of eggs-set at full maturity (HES, \%), pullet day-old chicks hatched at full maturity (PDC, \%), reject and unhatched day-old chicks at full maturity (RCU, \%). The different batches of each genotype, which were used as replicates, were reared under same management, with adequate feeding, nutrition, management and veterinary programme.

\section{Data classification and Analyses}

Data on the forty-four batches were grouped by genotype (22 batches for BN and 22 batches for IN) and by traits, while pullet weight at 20-weeks was classified into three namely: low $(<1599 \mathrm{~g})$, medium $(1600-1699 \mathrm{~g})$ and high $(>1700 \mathrm{~g}$ ) with replicates (batches) 14:18:12 respectively. The medium weight class was used as the standard for comparison. These data were subjected to General linear model (GLM) Factorial, LSM and ANOVA, $\alpha=0.05,0.01$ procedures. Mean separation was done using Tukey's HSD procedures, $\alpha=0.05$ were determined using Statistical Analytical Systems ${ }^{\circledR}$, v9.0 (SAS, 2002) computer software. Significant means were subjected to Cohen's difference (ds) classification for practical importance (Cohen, 1988). Study design adopted was randomized block design (RBD), while the linear statistical model was:

$\mathrm{Y}_{\mathrm{ij}}=\mu+\mathrm{G}_{\mathrm{i}}+\mathrm{T}_{\mathrm{j}}+(\mathrm{GT})_{\mathrm{ij}}+\varepsilon_{\mathrm{ijk}}$

Where,

$\mathrm{Y}_{\mathrm{ij}}=$ Observed response at full maturity in ith genotype, jth weight class and kth replicate.

$\mu=$ Overall and unknown mean

$\mathrm{Gi}=$ Fixed effect of $\mathrm{jth}$ genotype (i=Bovan nera, Isa brown)

$\mathrm{T}_{\mathrm{i}}=$ Fixed effect of $\mathrm{jth} 20$-week body weight class ( $\mathrm{j}=$ low, medium and high)

(GT)ij = Interaction effect of jth genotype and ith 20 -week body weight class.

$\varepsilon_{\mathrm{ij}}=$ Random error, assumed to be Normally and Independently Distributed $\operatorname{NID}\left(0, \sigma^{2}\right)$

The hypothesis of research was that genotype and 20-week pullet weight would have no significant effect on productive and hatching traits at full-sexual maturity. 


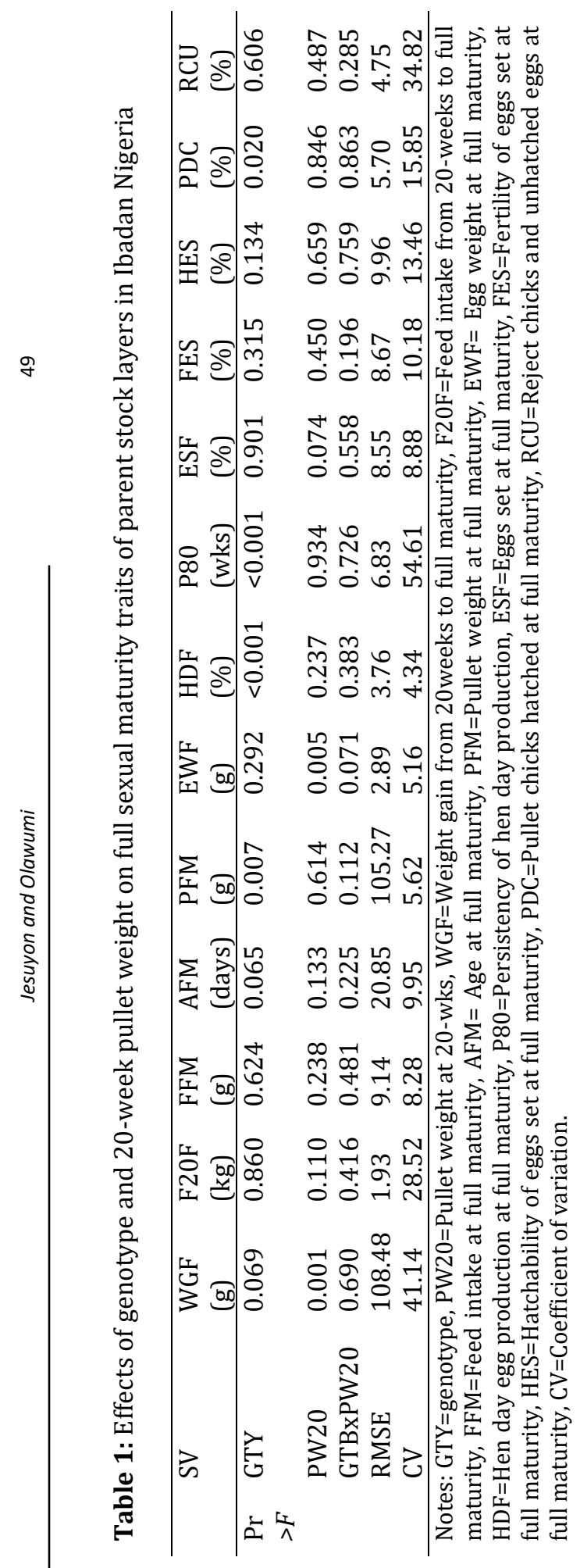




\section{RESULTS AND DISCUSSION}

Table 1 shows the effect of genotype and 20-week body weight on full sexual maturity traits in $B N$ and IB. Significant differences $(p<0.05)$ due to genotype were observed between BN and IB on PFM (g), HDF (\%), P80, (weeks) and PDC (\%). Among the three body weight classes at twenty weeks of age (PW20), pullet weight influenced weight gain to full maturity stage (WGF, g) and egg weight at full maturity (EWF, g). The interaction of GTYxPW20 produced major effects $(\mathrm{P}<0.05)$ on WGF (g), AFM (days), PFM (g), HDF (\%), P80 (weeks) and ESF (\%). Variability within traits was high on P80, WGF, RCU and F20F (CV=54.61-28.52), medium on PDC, HES and FES $(\mathrm{CV}=13.18-10.18)$ and low on AFM, ESF, FFM, PFM, and EWF, (CV=9.95-4.34).

\section{Effect of Genotype}

Table 2 shows significant genotypic differences $(\mathrm{p}<0.05)$ between $B N$ and IB on PFM (g), HDF (\%), P80 (weeks) and PDC (\%). BN had higher PFM while IB had higher HDF, P80 and PDC. AFM was higher in BN than IB by 13 days. Average PFM of BN was 0.88 SD greater than IB; but average HDF, P80, and PDC of IB were 1.28, 1.44 and 0.86 SDs more than BN respectively.

\section{Effect of 20-week pullet weight}

The 20-week pullet weight had effect $(\mathrm{p}<0.05)$ on WGF and EWF (Table $3)$. WGF was highest in the low $>$ medium $>$ high weight classes respectively. EWF was similar between low and medium weight classes, but was significantly lower than the high body-weight class. Standardized differences on WGF between low and medium weight classes (ds = 1.03); and on EWF between medium and high weight classes (ds $=1.19)$ were large and practical important for commercial breeding. Hatching traits (ESF, FES, HES, PDC and RCU, \%) between body weight classes were similar.

\section{Effect of Interaction between genotype and 20-week pullet weight}

Table 4 reveals significant interaction of GTY by PW20 classes. Within the low-weight class, BN was superior in PFM while IB was superior in P80 and ESF with important practical differences $(\mathrm{P}<0.05$; ds $=1.62,-1.59,-0.56)$. Medium-weight class revealed superiority of IB over BN ( $p<0.01, d s=-1.73$, 1.61) on HDF and P80. Within the high-weight class, BN was superior in WGF, but IB posted early AFM, superior HDF and P80 ( $\mathrm{p}<0.05$, ds=0.74, $-0.35,-1.03$ and -1.11). Within the low-weight class, BN produced higher EWF; but IB posted higher EWF in higher weight-classes $(P>0.05$, ds $=0.63,-0.72,-0.86)$. 


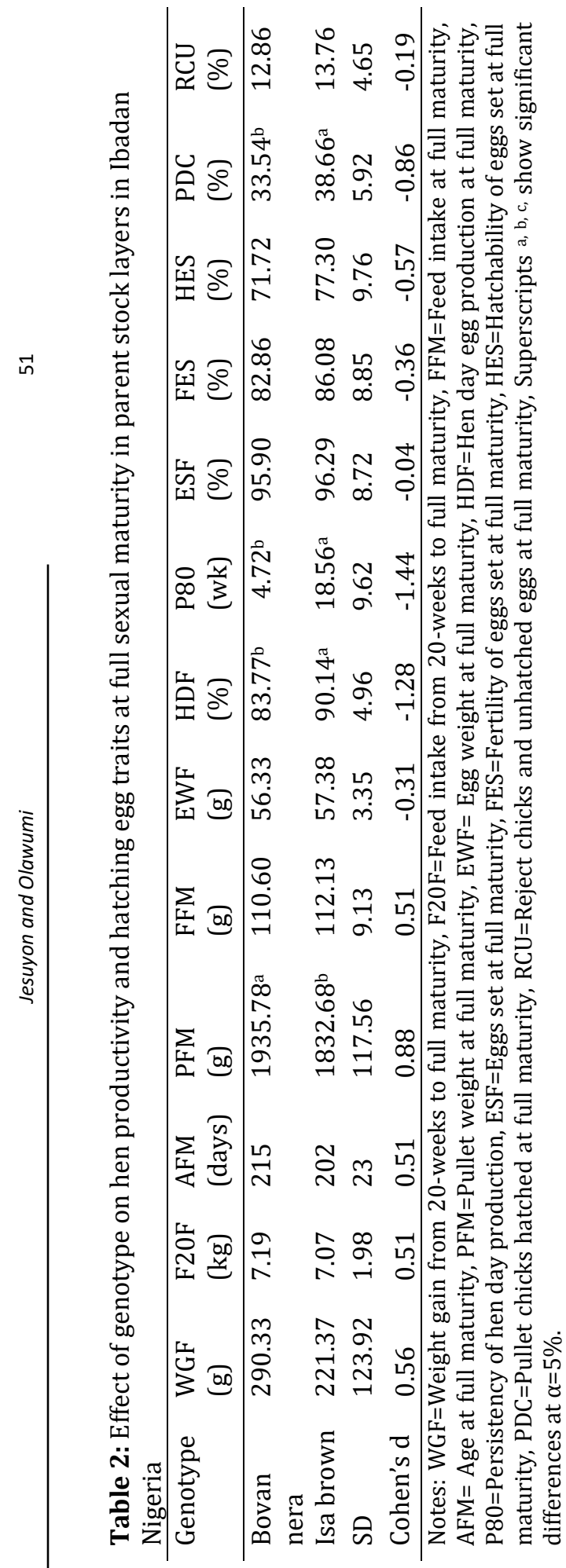




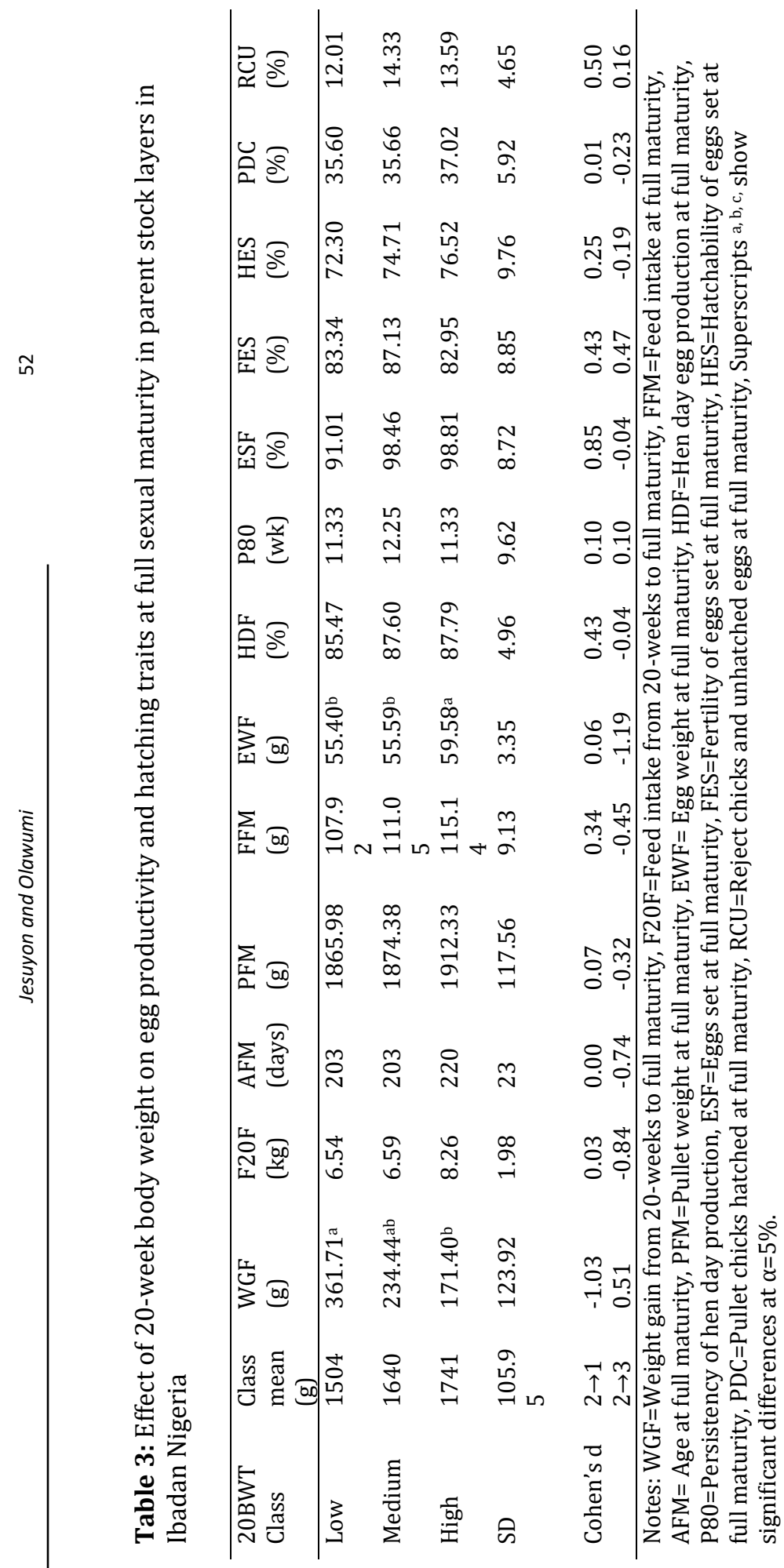




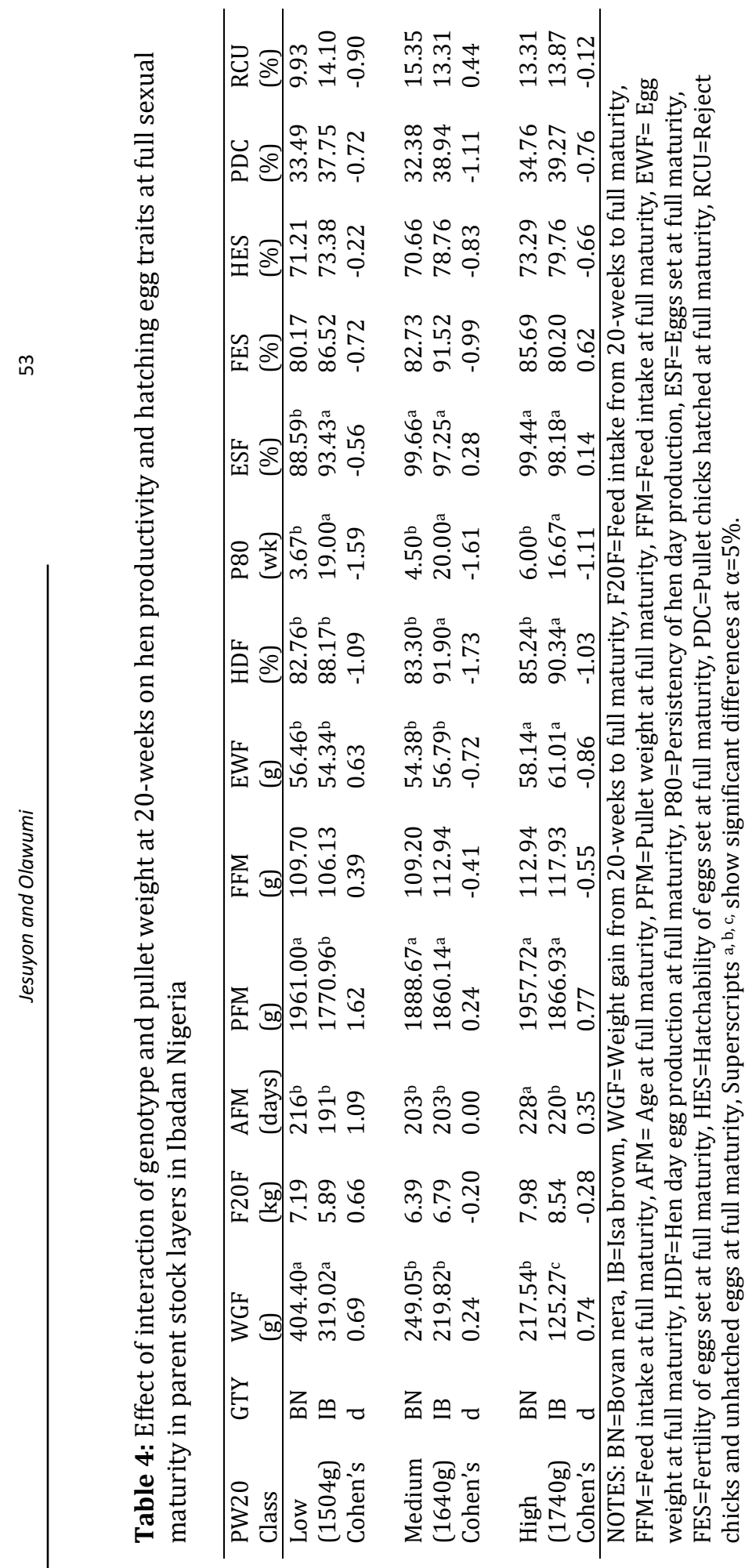


Between genotypes, the large standardized differences on PFM, HDF, P80 and PDC were important to practical breeding. Although BN was superior in body weight, IB was superior in hen-day egg production, persistency of egg production and percent pullet day-old chicks hatched, all at full maturity. These higher productivities of IB over BN could have been mediated by better body weight uniformity (due to early AFM, lower WGF, lower PFM) and higher genetic merit in HDF, P80 and PDC. Findings thus confirm previous reports on the superior genetics of IB over BN (Jesuyon, 2014).

The effect of 20-week weight class on WGF and EWF signify the importance of traits for chicken productivity and breed improvement. Weight gain difference between low and medium weight classes were large and revealed greater weight gain in the lower than medium weight class. Thus, better capacity for compensatory growth in the lower class than higher classes. Similarly, egg weight difference between medium and high weight classes was positive and large at FSM. The differences on EWT among weight categories $(0.00,0.19,4.18, \mathrm{~g})$ also revealed the higher impact of the high weight class, and supports literature on the highly positive correlation of body weight with egg weight (Oke et al., 2004). The longer interval (220 days) to FSM in the high weight class produced a nominal and positive improvement on HES and PDC $(\mathrm{P}>0.05)$ at FSM.

Interaction of GTY by PW20 classes discovered superiority of BN on PFM in low-weight class, and WGF in high-weight class respectively. This reveals that the higher the body weight at 20-weeks, the higher the WGF and thus rate of accretion of muscle, bone mass and weight gain until 32-34 weeks when the full mature body weight is achieved (Hyline, 2015). The relationship between body weight and egg weight must therefore be optimized for timely onset of peak egg production with high egg lay persistency, on high body weight for enhanced subsequent productivity in BN. The higher PFM of BN in low weight class and higher WGF in high weight class reveals BN's better genetics for growth. Similarly, IB was superior on $\operatorname{HDF}(5.41,8.60,5.10)$ and P80 (15.33, $15.5,10.67)$ in all weight classes, on ESF within the low-weight class, and posted early AFM in the high-weight class. Furthermore, IB was superior on ESF (5 \%) in low weight class, and early maturing (8 days) ability in high weight class. Thus, IB's better genetic ability for early maturity, high HDF, P80 and ESF commend it for adoption among tropical breeders. This superiority confirms previous reports of higher productive genetics of IB over BN (Jesuyon, 2014; Olawumi, 2014)

PFM obtained presently is within the weight range of $1840-1900 \mathrm{~g}$ reported on BN and IB (Olawumi, 2014); but higher than values 1.54, 1.55 and 
$1.64 \mathrm{~kg}$ reported on IB, BOVAN BROWN and Potchefstroom Koekoek reared under village production system (Tadesse et al., 2013). The EWF presently is higher than 51.45 and 51.35, g for crossbreds of Dominant black (DB) and Fulani ecotype (FE) chickens (DBxFE and FExDB) reported by Sola-Ojo and Ayorinde (2011); and 48.84g from Potchefstroom (Tadesse et al., 2013); but lower than 58.78 and 60.27, g from IB and BOVAN BROWN hens (Tadesse, 2013). Our findings contradict Render et al., 1984; and Ayorinde et al., 1988; that low body-weight were superior to high body-weight birds in egg production. Our results support Ayorinde and Oke (1995) who reported insignificant differences on egg production traits between heavy and low body weight birds; and Udeh (2007) who observed that rearing birds on weight basis may have no significant advantage, because high and light body weight birds reared in same environment recorded similar results in hen-day egg production within breed. Present fertility and hatchability values are above 74.89-84.08, \% and 65.62-78.56, \% from Nigerian indigenous chickens (Ajayi and Agaviezor, 2016). Similarly, while FES is lower than 91.1 and 94.5, \%, HES is higher than 64.0 and $80.6, \%$ on Rhode Island and crossbred chickens respectively raised in Tanzania (Malago and Baitilwake, 2009).

\section{CONCLUSION}

ISA BROWN presented better genetics for early maturity, hen-day egg production, persistency of egg production, and percent pullet day-old chicks hatched at full sexual maturity. Low weight flocks demonstrated better compensatory growth from 20th week to full sexual maturity, while high body weight flock laid bigger eggs. GTY by PW20 interaction revealed that farmers could rear either genotype to 20 -week body weight of $\geq 1640 \mathrm{~g}$ to obtain close to optimum genetic productivity at full sexual maturity.

\section{ACKNOWLEDGEMENTS}

The authors wish to express appreciation to the management and staff of CHI farms limited, Ibadan Nigeria, for data used for the study.

\section{REFERENCES}

Ajayi, F.O., Agaviezor, B.0. 2016. Fertility and hatchability performance of pure and crossbred indigenous chicken strains in the high rainforest zone of Nigeria. Intl $\mathrm{J}$ of Livestock Prod. 7, 12, 141-144. DOI: 10.5897/IJLP2016.0308.

Ayorinde, K.L., Toye A.A., Aruleba, O.A. 1988. Association between body 
weight and some egg production traits in a strain of commercial layers. Nig J Anim Prod. 15, 119-121.

Ayorinde, K.L., Oke, U.K. 1995. The influence of juvenile body weight and two feeding regimes during the growing phase on growth performance and early lay characteristics of pullets. Nig J Anim Prod. 22, 101-107.

Connie, L., Bish, W. L., Beane, P. L., Ruszler, J.A.C. 1985. Body weight influence on egg production. Poult Sci. 64, 2259-2262.

Cohen, J. 1988. Statistical power analysis for the behavioural sciences. Second Edition, Lawrence Earlbaum Associates, Hillsdale, NJ.

Hyline, 2015. Hy-line brown commercial management guide. Page 18. https//www.hyline.com

Duncan, J. H., Mench, J. 2000. Does hunger hurt letter. Poult. Sci. 49, 934.

Jesuyon, O. M. A. 2014. Multivariate analysis of production variables of layer chickens on deep-litter in the humid tropics. STOČARSTVO. 68 (1) 1923. UDK 636. 083.14

Haq, R., Haq, E., Khan, M. F. 2011. Correlation between body weight and egg weigh of Dokki and Fayoumi hens in Pakistan. J. Basic and Appl. Sci., 7: 165-168

Malago, J. J., Baitilwake, M. A. 2009. Egg traits, fertility, hatchability and chick survivability of Rhode Island Red, local and crossbred chickens. Tanzania Veterinary Journal. 26, 1, 24-36.

Oke, U.K., Herbert, U., Nwachukwu, E.N. 2004. Association between body weight and some egg production traits in the guinea fowl (Numida meleagris galeata). Livestock Res for Rural Dev. 16, No. 72. DOI: http://www.lrrd.org/lrrd16/9/oke16072.htm

Olawumi, S.O. 2011. Study on pre-laying characteristics of three breeds of commercial layers in the derived savannah zone of Nigeria. Pakistan J Biol Sci. 14, 23, 1061-1065. DOI: 10.3923/pjbs.2011.1061.1065

Olawumi, S.O., 2014. Egg production of Isa Brown and Bovan Nera laying hens as affected by body weight during one-year laying cycle. International Journal of Applied Poultry Research, 3, 1, 4-7.

Olawumi, S. 0. 2014. Effect of short-term feed restriction on production traits of brown and black plumage commercial layer strains at late phase of egg production. American J. Agric. And Forestry, 2(2): 33-38.

Render, J.A., McDaniel, G.R., Mcquire, J.A. 1984. Egg characteristics and egg production efficiency of dwarf white leghorn hens divergently selected for body weight. Poult Sci. 63, 214-219.

SAS 2002. Statistical Analytical systems (SAS 9.0) software.

Sola-Ojo, F.E., Ayorinde, K.L. 2011. Evaluation of reproductive performance and egg quality traits in progenies of dominant black strain crossed with Fulani ecotype chicken. J Agric Sci. 3, 1, 258-265.

Tadesse, D., Singh, H., Mengistu, A., Esatu, W., Dessie, T. 2013. Study on 
productive performances and egg quality traits of exotic chickens under village production system in East Shewa, Ethiopia. Afric J Agric Res, 8, 13, 1123-1128. DOI: 10.5897/AJAR2013.6987

Tesfaye, E., Haile, A., Dessie, T. 2009. Effects of feed restriction on production and reproductive performance of Rhode Island red pullets. Afric J of Agric Res. 4, 7, 643-648.

Udeh, I. 2007. Influence of weight grouping on the short-term egg production of two strains of layer type chicken. Anim Res Intl. 4, 741-744. 